\title{
Abstracts of Theses Approved for the M.Sc. Degree at the Health Sciences Centre, University of Kuwait
}

\section{Typing of Helicobacter pylori VacA and CagA Genotypes in Relation to Histological Gastric Lesions in Kuwait}

\author{
A.A. Al-Qabandi \\ Department of Pathology, Faculty of Medicine, \\ Kuwait University, Kuwait
}

Objectives: To assess the relation between Helicobacter pylori CagA and vacuolating cytotoxin (VacA) types, the disease patterns and cytokine secretion in patients in Kuwait. Materials and Methods: Biopsy specimens were obtained from 117 patients undergoing routine upper gastrointestinal endoscopic procedures. Three standard size pinch biopsies were taken from the antrum using Tentax and Olympus endoscopies, one of which was used for histological assessment, the second for PCR studies, and the third for cytokine ELISA. Formalinfixed, paraffin-embedded gastric tissue sections were stained routinely with HE, AB/PAS and silver HPSS stains. The severity of gastritis, density of HP, activity, atrophy and metaplasia were graded using the Sydney system and correlated with the HP subtypes. PCR was used for HP typing. ELISA was used for quantitative measurement of different cytokines that are induced by the organism. Cytokine production was also correlated with different HP subtypes. Results: Of the 117 endoscopic biopsies studied, 109 (93.2\%) had varying grades of gastritis. Eight (6.8\%) showed no inflammation. Of 117 biopsies, HP was not detected in $19(16.2 \%)$. Of 117 biopsies, $52(44.4 \%)$ were CagA-positive and $65(55.6 \%)$ CagA-negative, while $93(79.4 \%)$ were VacA-positive and $24(20.5 \%)$ were VacA-negative. When CagA genotype was correlated with histological and endoscopic findings, the results showed that CagA-positive samples had higher scores than CagA-negative ones for gastritis ( 96 vs. $91 \%$ ), activity ( 81 vs. 65\%), atrophy (77 vs. $55 \%$ ), metaplasia (19 vs. $16 \%$ ) and DU (71 vs. $42 \%$ ). When VacA genotype was correlated with histological and endoscopic findings, the results showed that VacA-positive samples had higher scores than VacA-negative ones for gastritis (97 vs. $79 \%$ ), activity ( 80 vs. $42 \%$ ), atrophy (74 vs. $29 \%)$, metaplasia (18 vs. $12 \%$ ) and DU (62 vs. $25 \%$ ). IL-8 was higher in HP-positive samples than in HP-negative ones. IL-8 concentrations were higher in CagA-positive samples compared to all other HP subtypes. IL- 6 could not be detected in 50 out of a total of 99 specimens analyzed. IL-6 levels showed no association with HP status. Conclusion: In this study, CagA and VacA correlated well with inflammation severity, atrophy and DU $(p<0.01)$. There was significant correlation between IL-8 and DU, atrophy, activity and inflam- mation severity $(\mathrm{p}<0.001)$. IL-8 also correlated well with CagA status $(\mathrm{p}<0.05)$. However no significant correlation was found between IL-6 and endoscopic or histological or CagA and VacA status.

T.A. Junaid (Supervisor), Department of Pathology

A.S. Mustafa (Co-supervisor), Department of Microbiology

\section{Genetic Polymorphisms Conferring Resistance against HIV-1 Infection and AIDS: Frequency in Kuwait and Genotyping of HIV-1-Positive Kuwaitis}

M.B. Al-Turab

Department of Microbiology, Faculty of Medicine, Kuwait University, Kuwait

Objectives: To estimate the frequencies of 59029-G and 59029A alleles in healthy Kuwaitis and to genotype HIV-infected Kuwaitis for mutations affecting the pace of progression to AIDS, namely: CCR5-432, CCR5-m303, 59029-G/A, CCR2-64I and SDF1-3'A. Materials and Methods: The DNA was extracted from leukocytes of 109 healthy unrelated Kuwaitis, and from 27 HIV-infected Kuwaitis by using a 'salting out' method. Genotyping of CCR5- $\Delta 32, \mathrm{~m}-303$, 59029-G/A, CCR2-64I and SDF1-3'A was performed by PCR/RFLP tests. The fragments obtained after digestion with restriction endonucleases were sized by agarose gel electrophoresis. Results: The frequencies of 59029-G and 59029-A alleles among 109 healthy individuals genotyped were 0.66 (95\% CI 0.59-0.72) and 0.34 (95\% CI 0.28 0.41 ), respectively. None of $27 \mathrm{HIV}$-infected Kuwaitis carried the resistance alleles for the CCR5- $\Delta 32$ and m-303 mutations. The AIDS resistance genotypes were identified in 2 (homozygosity for SDF1-3'A allele), 9 (heterozygosity for CCR2-64I allele) and 12 (homozygosity for 59029-G allele) patients out of 27 investigated. Nine combinations of genotypes potentially conferring various degrees of resistance to AIDS were identified. Conclusion: The frequency of the AIDS protective allele 59029-G in healthy Kuwaitis is significantly higher than the frequency of 59029-A allele and is among the highest reported by now. Prospective clinical studies are required for establishing the prognostic values of different AIDS protective genotypes and their combinations in HIV-positive Kuwaitis.

A. Voevodin (Supervisor), Department of Microbiology

\section{KARGER}

Fax +41613061234

E-Mail karger@karger.ch

www. karger.com 